\title{
Reflectometry for Wendelstein 7-X
}

\author{
M. Hirsch, E. Holzhauer*, H.-J. Hartfuss \\ Max-Planck-Institut für Plasmaphysik, EURATOM Association, Greifswald, Germany \\ *Institut für Plasmaforschung, Universität Stuttgart, Stuttgart, Germany
}

The fully optimized stellarator Wendelstein 7 - X, now under construction in Greifswald, Germany, explores stellarator optimization and high-performance steady-state operation. In a first stage $\mathrm{P}_{\mathrm{ECRH}}=10 \mathrm{MW}$ for $30 \mathrm{~min}$. planned. The diagnostic equipment (Hartfuss et al 1996) involves a reflectometry system dedicated to measure edge density profiles and to characterize density perturbations and their poloidal propagation velocity. Preparatory work such as design activities and the installation of a single antenna pair are included in the set of the so-called start-up diagnostics. First plasma operation is expected early 2012.

The position of the main reflectometer planned (Fig.1) is chosen such to minimize the curvature of the probed flux surfaces and where the B-field gradient increases continuously along the line-of sight like in a tokamak. The latter gives best access to the plasma core in $\mathrm{x}$ mode either via the right-hand cut-off with low-field-side launch or via the left-hand cut-off launching at the same toroidal position from the high-field-side. In standard operation the local on-axis magnetic field is $\mathrm{B}=2.59 \mathrm{~T}$ with a variation of $2.35 \mathrm{~T}<\mathrm{B}<2.94 \mathrm{~T}$ along the sightline. Due to the strongly elongated plasma shape and the nearly vertical $\mathrm{B}_{\text {mod }}$ surfaces, the $\mathrm{x}$-mode cut-off in the vicinity of the equatorial plane can be well approximated by the flux surfaces. Their poloidal curvature is low and varies as $80 \mathrm{~cm}<R_{\text {curv }}<100 \mathrm{~cm}$ with cut-off layers $r / a>0.5$. The toroidal curvature can be neglected. The variation of the conditions for reflectometry within the set of standard configurations of the stellarator vacuum magnetic field (Andreeva 2002) is small and neglected at this state of the design.

Expected cut-offs and resonances are given on Fig.2. For this design a density profile with central density $n(0)=1.10^{20} \mathrm{~m}^{-3}$ and steep edge gradients is estimated based on W7-AS results. A reference cut-off layer is assumed to be at $(\mathrm{r}-\mathrm{a})=-3 \mathrm{~cm}$ in effective magnetic coordinates equivalent to $\mathrm{r} / \mathrm{a}=0.94$ where the average minor radius $a=52 \mathrm{~cm}$ corresponding to the position of maximum velocity shear in an H-mode edge layer. Due to the elliptical plasma cross section the flux compression within the equatorial plane is about a factor of 2 such that one obtains $\Delta R \cong \Delta r_{\text {eff }} / 2$. Correspondingly the reference cut-off layer is located only $1.5 \mathrm{~cm}$ inside the separatrix. For the reference density profile this layer yields the x-mode cut-off for a frequency $f=90 \mathrm{GHz}$ (center of the W-band) probing a density of $2.610^{19} \mathrm{~m}^{-3}$. 


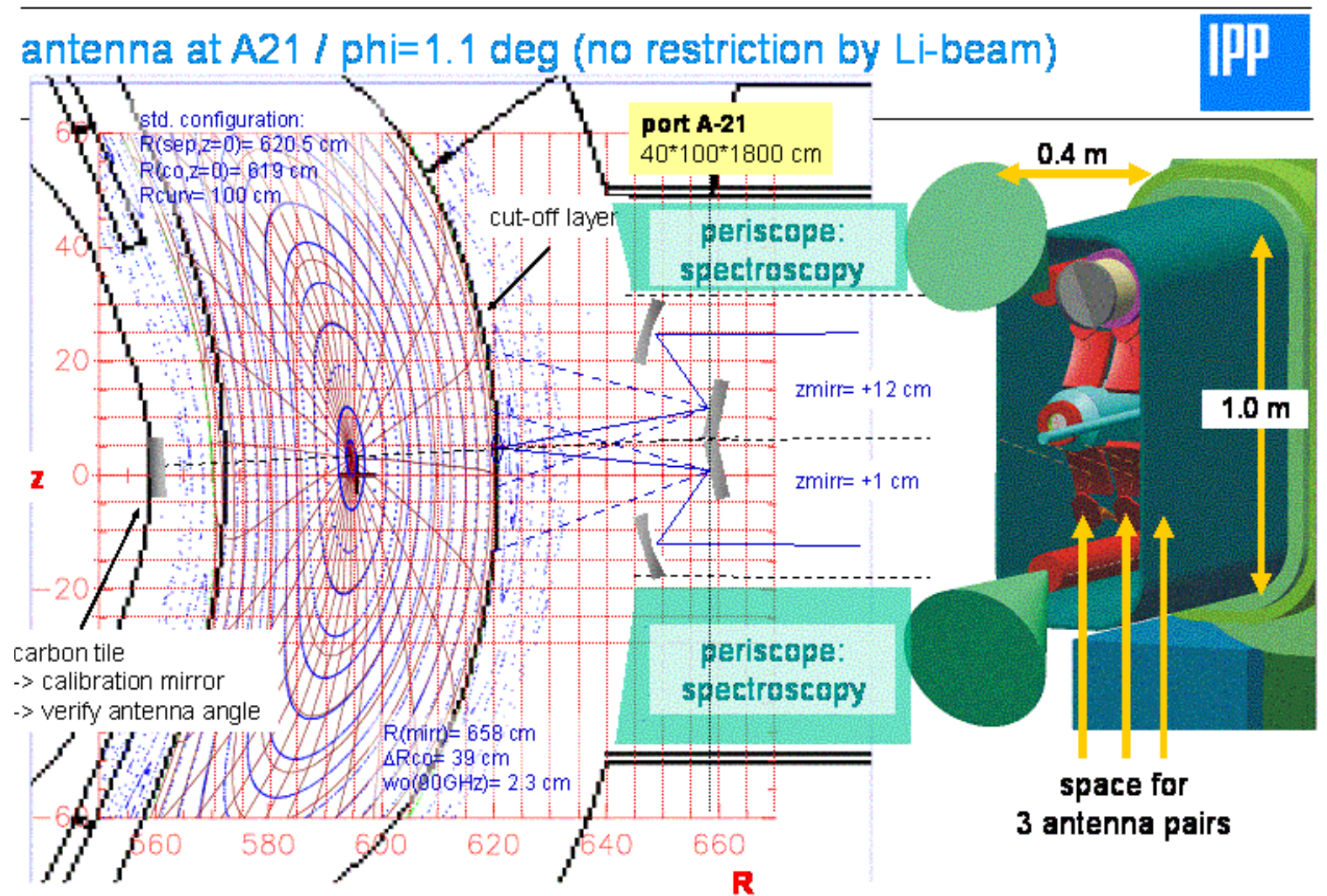

M Hirsch, IRWT, Garching 2005, p. 6

Fig.1: Flux surfaces (standard magnetic configuration) and plasma vessel contours at the diagnostic position $\varphi=0, \mathrm{z}=0$. The port AEA21 (artists view on the right side) is shared with a variety of other diagnostics such as spectroscopy periscopes.

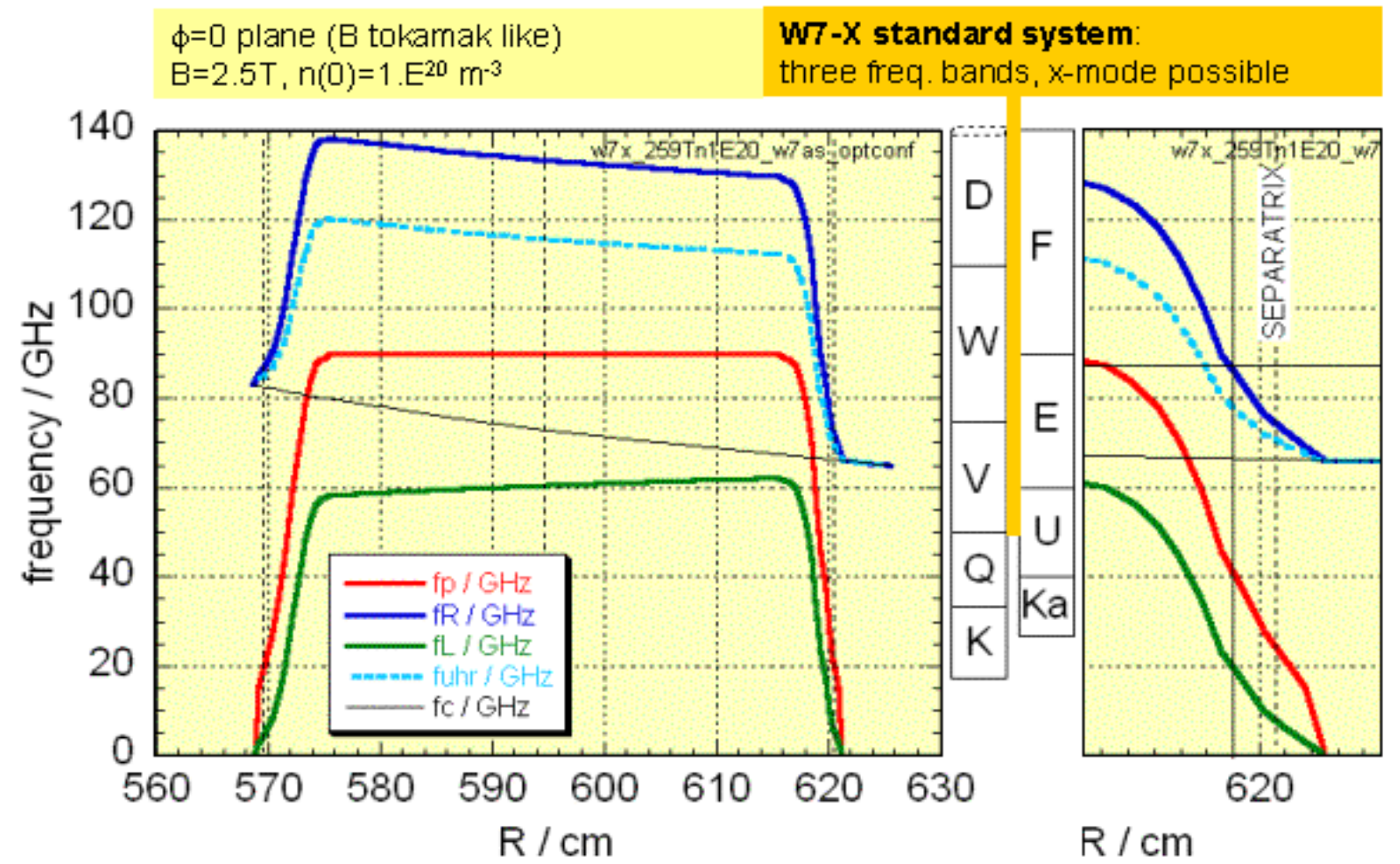

Fig. 2 Cut-offs and resonances along the reflectometry sightline $(\varphi=0, z=0)$ for a design density profile with $\mathrm{n}(0)=1.10^{20} \mathrm{~m}^{-3}$. 
The diagnostic port AEA21 allows for three pairs of toroidally neighboured antenna pairs each with focussing elliptical mirrors with maximum diameter of 10 to $12 \mathrm{~cm}$. Directly opposite on the high-field side carbon covered tiles at the inner vessel wall are designed as microwave mirrors (surface $10 \times 10 \mathrm{~cm}^{2}$ ) in order to calibrate the time delay measurements (Fig.3). Thermal effects on their radial position are small: If during operation the plasma vessel heats up from 20 to $60 \mathrm{C}^{\circ}$ the tiles move radially outward by about $2.4 \mathrm{~mm}$. The launching mirror positions are expected to remain almost unchanged as they are fastened to the flange which is assumed to stay at room temperature. These reflector tiles also would allow for an integrated HFS antenna - however with very restricted space available (about $3 \mathrm{~cm}$ to the vessel wall) (see Fig.3, left).

One of the antenna systems is planned to operate in an early state of W7-X operation. A high-directivity dual-antenna arrangement is proposed where the tilt angle of both sightlines with respect to the cut-off layer $\theta_{\text {tilt }}$ can be varied independently to provide flexibility. Highdirectivity can well be realized by a Gaussian antenna characteristic with nearly parallel wave fronts and a spot size taking into account the curvature of the reflecting laye: The latter yields a compromize between maximum number of perturbation maxima illuminated and minimum curvature within the spot (Hirsch and Holzhauer 2004). For example with $f=90 \mathrm{GHz}$ probing a density of $2.610^{19} \mathrm{~m}^{-3}$ in $\mathrm{x}$-mode and a tilt $\theta_{\text {tilt }}=13.8 \mathrm{deg}$ which selects perturbations $K_{\perp}=9$ $\mathrm{cm}-1$ the calculated optimum beam width is $w_{o}^{o p t}=2.3 \mathrm{~cm}$ (1/e-decay width of the E-field amplitude) resulting in a resolution of $\left(\Delta K_{\perp} / K_{\perp}\right)^{-1}=5.2$. The optimum beam waist varies as $w_{o}^{o p t} \propto \sqrt{\rho \cdot \lambda}$ where $\rho$ is the effective curvature radius and thus inherently fulfils the condition $w_{o}(\lambda) \propto \sqrt{\lambda}$ which allows for a broadband Gaussian optics with arbitrary mirror positions (Hirsch and Holzhauer 2005). A lower boundary $f>70 \mathrm{GHz}$ arises only from the finite aperture which can be realized for the focussing mirrors within the port environment. To guide the beam in the long port to the vacuum flange a quasioptical transmission line is planned which also has the advantage that there is no need for cooling waveguides. The variable antenna orientation is achieved by a steerable plane first mirror which decouples the sightline geometry in particular the tilt angle - from the optimization of the spot size resulting from the second fixed elliptical mirror. Here the $\theta_{\text {tilt }}$ defines the selected density perturbations $K_{\perp}$ via the Bragg condition whereas the spot size determines the spectral resolution $\Delta K$. The steerable antenna sightlines allow for a number of different arrangements: a) Density profile measurements with separate antennas for signal launch and reception oriented to a common beam spot (bistatic arrangement), b) conventional poloidal correlation reflectometry where the two antennas are each oriented perpendicular to the cut-off layer probing long-wavelength perturbations such as MHD, c) Doppler reflectometry for a variety of different tilt angles $\theta_{\text {tilt }}$ with the two sightlines oriented in opposite poloidal directions such that the frequency shifts resulting from varying $\theta_{\text {tilt }}$ (induced by varying configuration or by MHD) can be separated from the Doppler shift resulting from a variation of the propagation velocity of the perturbations $\mathrm{v}_{\perp}$ (Hirsch and Holzhauer 2004). As two poloidally separate spots are probed this arrangement can be used for poloidal correlation Doppler reflectometry simultaneously. Poloidal correlation Doppler reflectometry measures the poloidal correlation length of small scale (finite $K_{\perp}$ ) perturbations and delivers a time-of-flight measurement which is the complementary method to determine the poloidal propagation velocity $\mathrm{v}_{\perp}$ (Hirsch and Holzhauer 2004). d) The $K_{\perp}$-spectrum of turbulence is obtained with the proposed system by means of a temporal scan of $\theta_{\text {tilt }}$ (see Hirsch 
et al 2001, Hennequin et al 2005). Finally a fast monitoring of the $K_{\perp}$-spectrum is possible by simultaneously tracking the signal with two different values of $\theta_{\text {tilt }}$ which is of interest in the context of fast changes of the $K_{\perp}$-spectrum induced by shear flow for example. Limitations for the accessible $\theta_{\text {tilt }}$ arise from shadowing and crosstalk between the two antenna patterns involved.

\section{integrated HFS-antenna: possible port access via AEC41}

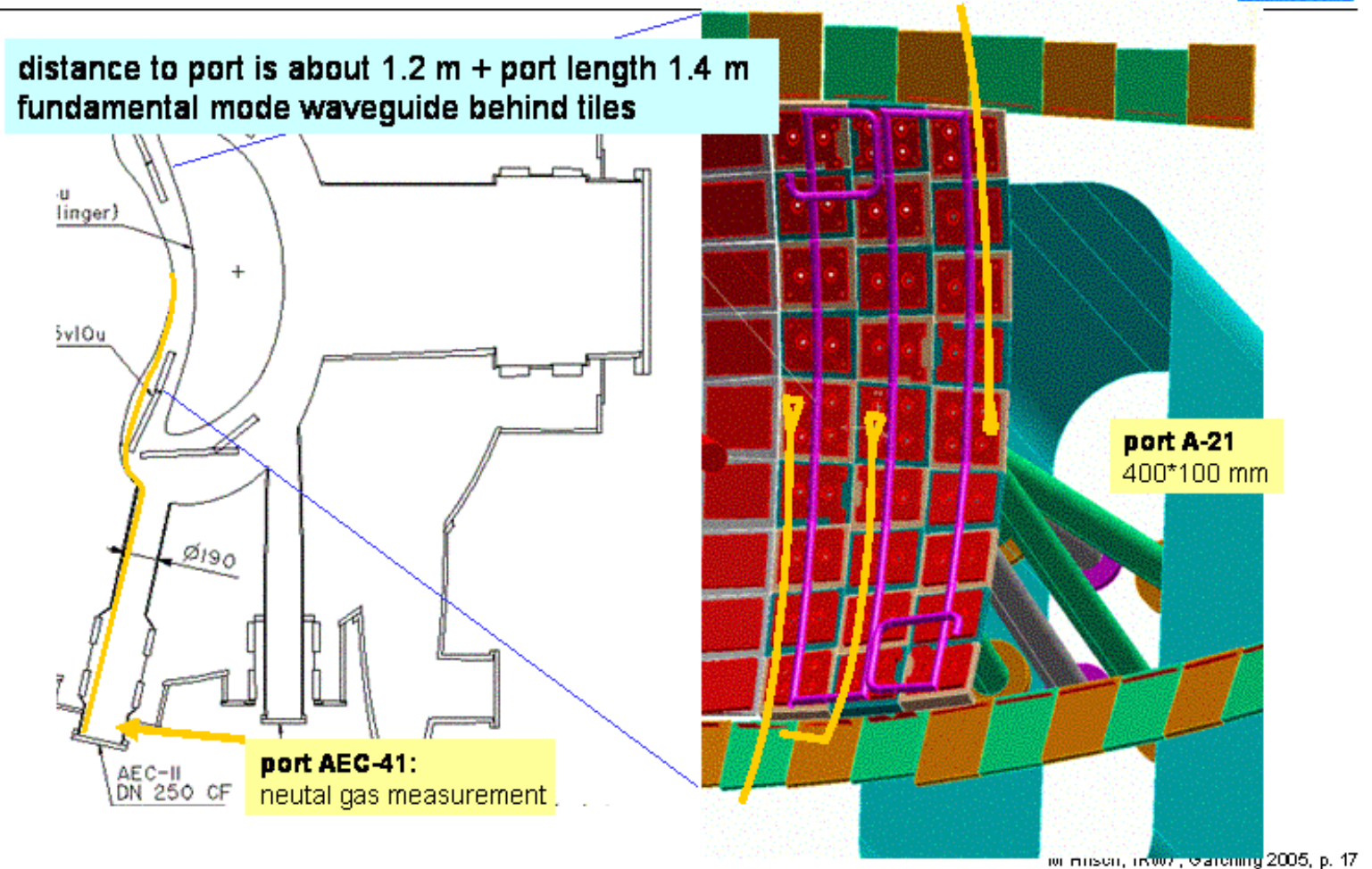

Fig. 3: Possible geometry for integration of a HFS antenna in the first-wall high-heatloads tiles (200 to $250 \mathrm{~kW} / \mathrm{m}^{2}$ ). The corresponding waveguides leading towards the next port distance to the port $1.2 \mathrm{~m}$, portlength $1.4 \mathrm{~m}$ are indicated in yellow. In the right figure the tiles are shown from the high-field side (vessel wall omitted), $\mathrm{CuCrZr}$ cooling bodies in red, cooling pipes in purple. The graphite surface of the tiles right opposite the three antenna pairs is oriented as calibration mirrors for the time-of-flight measurement.

The antennas must withstand a heat-load of about $50 \mathrm{~kW} / \mathrm{m}^{2}$ which requires active cooling. The level of microwave stray radiation resulting from non-absorbed $140 \mathrm{GHz}$ ECRH heating power is expected up to $100 \mathrm{~kW} / \mathrm{m}^{2}$ near the ECRH launching planes depending on heating scenarios and the position in the vessel considered (The reflectometry antennas are toroidally $72 \mathrm{deg}$ apart from the ECRH launching plane). All in-vessel parts will be tested under these conditions in a test-chamber (Ullrich S. et al 2005).

Outside the vessel a bundle of oversized quasioptical transmission lines are planned to guide the microwave signals to the diagnostic cabin right outside the bioshield (Fig.4). The single-path length is about $27 \mathrm{~m}, 5$ mitre bends are necessary to account for radiation shielding and space requirements in the torus hall. In the diagnostic cabin quasi-optical beam splitters (Simonetto et al 2004) will offer the flexibility to adapt different transmitter/receiver 
systems and use the full spectrum of reflectometry measurements.

\section{reflectometry transmission line}

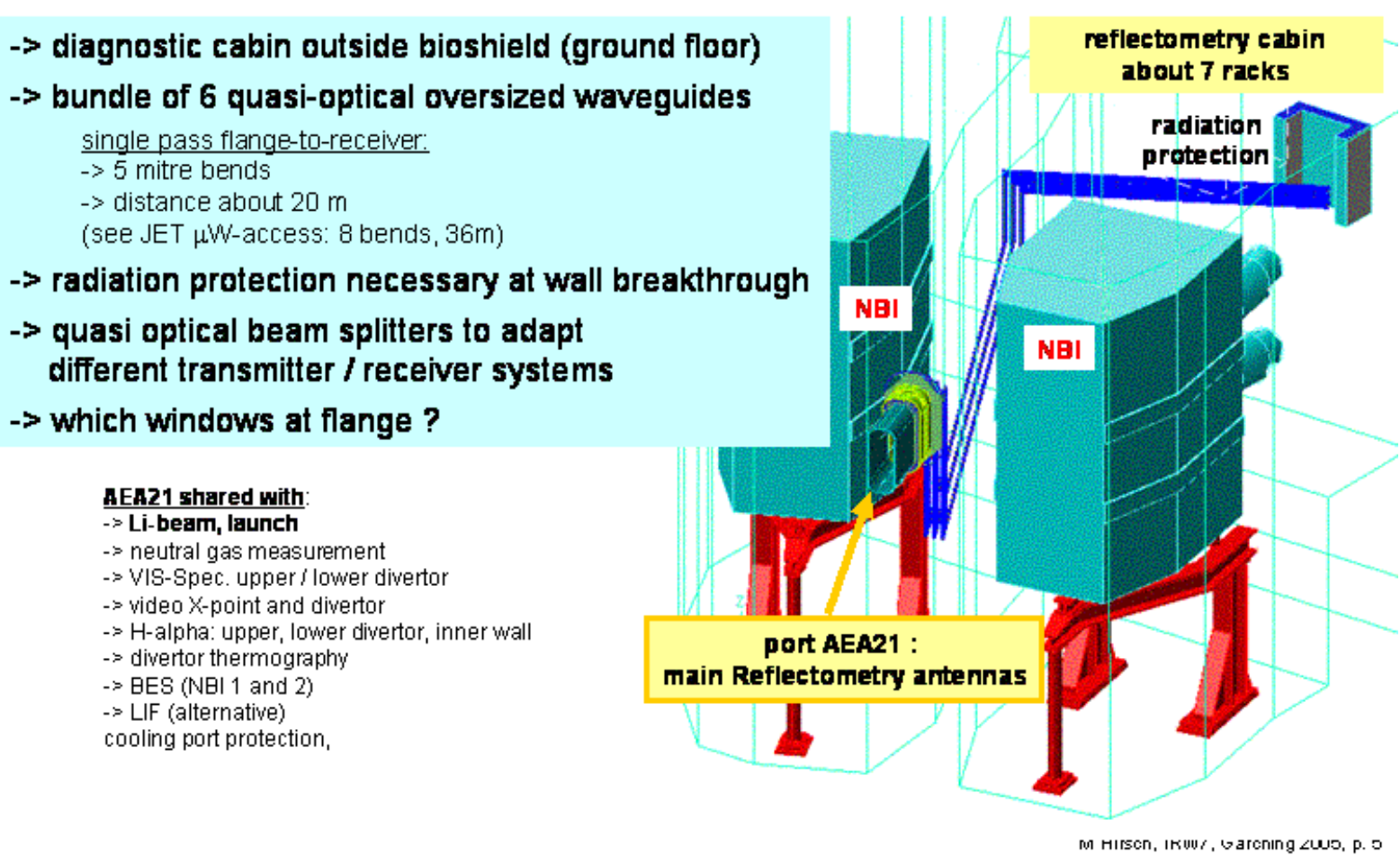

Fig.4 Transmission lines to port AEA21. The bioshield (concrete with thickness $1.8 \mathrm{~m}$ ) is omitted. From the diagnostic cabin outside only the radiation protection is shown.

\section{REFERENCES}

Hartfuss H.-J. et al, Rev. Sci. Instrum 68, 1244 (1996).

Andreeva T., IPP Report III/270, May 2002.

Hirsch M. and Holzhauer E. to be submitted to Nuclear Fusion 2005

Hirsch M. and Holzhauer E. Plasma Phys. Control Fusion 46 (2004) 593-609

Hirsch M. et al Plasma Phys. Control Fusion 43 (2001) 1641-1660

Hennequin P. et al this workshop

Ullrich S. Diploma Thesis University of Greifswald, summary in Stellarator News 98, May 2005 available on the web: http://www.ornl.gov/sci/fed/stelnews/

Simonetto A. et al, Design of the Quasi Optical Interface System for JET's new Microwave Access, Proc. of 2004 29th Int. Conf. on Infrared and Millimeter Waves Karlsruhe, Germany, Sept 27-Oct 1, 2004, p 705, edt. by M. Thumm and W. Wiesbeck 